\section{August 2009}

27.-29.08.2009

13. Fachgruppentagung Rechtspsychologie der Deutschen Gesellschaft für Psychologie (DGPs): "Auf der Suche nach der Wahrheit" Information: http://www.uni-giessen. $\mathrm{de} / \mathrm{cms} / \mathrm{fbz} / \mathrm{fb} 06 /$ psychologie/abteilungen/ sozial-und-rechtspsychologie-1/ rechtspsychologietagung09

\section{September 2009}

2.-5.09.2009, Sorrento, Italien

19th Conference

European Association of Psychology

and Law (EAPL)

Information: www.sara-cesvis.org

07.-11.09.2009, Königslutter

Curriculum Forensische Psychiatrie und Psychologie

Psychiatrisch-psychologische Begutachtung

im Strafverfahren, Teil 1

Prof. Dr. N. Konrad, Dr. Baltzer, Dipl.-Psych.

Dr. Herbig

Information: www.psychiatrie-akademie.de

17.-19.09, Gießen

11. Tagung der Kriminologischen

Gesellschaft Wirtschaftskriminalität

- Gewalt - Aktuelle Erscheinungsformen

und Prävention

Information: www.krimg.de/drupal/node/3

23.-24.09.2009, Leipzig

Provozieren - Pöbeln - Zuschlagen : Was

steckt hinter Jugendgewalt ?

Die Kinderschutz-Zentren

Information: www.kinderschutz-zentren.

org/leipzig2009

$$
\text { Oktober } 2009
$$

08.-10.10.2009, München

24. Herbsttagung für Forensische Psychiatrie Prof. Dr. N. Nedopil

Abteilung für Forensische Psychiatrie der

Universität München

Information: www.forensik-muenchen.de

28.-30.10.2009, Solingen

Lockerungsentscheidungen

im Maßregelvollzug und

Gefährlichkeitsprognosen

Prof. Dr. N. Leygraf, Dipl.-Psych. K. Elsner

Information: www.rips.lvr.de

29.10.-01.11.2009, Baltimore Marriott

Waterfront, USA

40. Annual Meeting

American Academy of Psychiatry and the

Law

Information: www.AAPL.org

\section{November 2009}

11.-14. November 2009, Philadelphia, USA Annual Meeting

American Society of Criminology

Information: http://www.asc41.com/

annualmeeting.htm

\section{März 2010}

17.-20.03.2010, Vancouver (Canada)

Conference of the American Psychology-Law Society

Westin Bayshore Hotel

American Psychology-Law Society (Section 41

of the APA)

Information: www.ap-ls.org

\section{April 2010}

12.-14.04.2009, Potsdam-Griebnitzsee 13. Basiskurs für Forensische Psychiatrie Prof. Dr. H.-L. Kröber

Institut für Forensische Psychiatrie der Charité Information: www.forensik-berlin.de

12.-15.04.2010, München

15. Bundeskongress der Psychologinnen und Psychologen im Justizvollzugs

Generalisten und Spezialisten - Facetten

einer Profession

Arbeitskreis der Psychologen im bayerischen Justizvollzug e.V. zusammen mit dem Department Psychologie der LudwigMaximilians-Universität München. Information: www.buko-vollzugspsychologen. de

\section{Mai 2010}

25.-27.05.2010, Vancouver (Canada)

10th Annual IAFMHS Conference

Westin Bayshore Hotel

International Association of Forensic Mental Health Services (IAFMHS)

Information: www.iafmhs.org

\section{Juni 2010}

11.06.2010, Berlin-Dahlem

14. Berliner Junitagung für Forensische Psychiatrie und Psychologie

Institut für Forensische Psychiatrie der Charité Information: www.forensik-berlin.de (Veranstaltungen)

15.-18.06.2009, Göteborg, Schweden 20th Conference: Towards a positive legal psychology

European Association of Psychology and Law (EAPL)

Information: http://eapl2010.net/ 